\title{
Genetic analyses of chromosome 12 loci in Crohn's disease
}

Fondation Jean Dausset/CEPH and Unité INSERM 434, 27 rue Juliette Dodu,

75010 Paris, France

$S$ Lesage

H Zouali

M Chamaillard

I Le Gall

G Thomas

J-P Hugot

EPIMAD, Registre des Maladies

Inflammatoires du

Tube Digestif du Nord

Ouest de la France,

Unit of Epidemiology,

CHRU de Lille, France

J-F Colombel

GETAID Groupe

d'Etudes

Thérapeutiques des

Affections

Inflammatoires

Digestives, $\mathrm{CHU}$ de

Liège, Belgium

J Belaiche

European Pediatric

Group on the Genetics of IBD (EPGWGIBD),

Hôpital Robert Debré,

Paris, France

J-P Cézard

J-P Hugot

Örebro Medical

Centre, Örebro,

Sweden

C Tysk

University Hospital, Linköping, Sweden S Almer

Hospital Universitari Germans Trias I Pujol,

Badalona, Spain

M Gassull

Herlev Hospital, Herlev, Denmark V Binder

Hôpital Saint-Antoine, Paris, France

G Thomas

All authors (except ILG) are associated with the

"European Concerted Action on the Genetics of Inflammatory Bowel Diseases".

Correspondence to: Dr J-P Hugot, Fondation Jean Dausset/CEPH, 27, rue Juliette Dodu, 75010 Paris, France.

jean-pierre.hugot@cephb.fr

Accepted for publication 23 May 2000

S Lesage, H Zouali, J-F Colombel and EPIMAD, J Belaiche and GETAID, J-P Cézard and EPGWGIBD, C Tysk, S Almer, M Gassull, V Binder, M Chamaillard, I Le Gall, G Thomas, J-P Hugot

Background and aims-Inflammatory bowel disease (IBD) includes ulcerative colitis and Crohn's disease, both of which are multifactorial diseases involving the interaction of genetic and environmental factors. A region on chromosome 12 centred around the marker locus D12S83 has previously been associated with IBD predisposition. The aim of the study was to investigate this genetic region in an independent panel of European families affected by Crohn's disease.

Methods-A sample of 95 families with two or more affected relatives and 75 simplex nuclear families were genotyped for 19 microsatellite loci located on chromosome 12. A search for linkage and linkage disequilibrium was performed using nonparametric two point and multipoint analyses with the Analyze and Genehunter packages.

Results-No evidence of linkage or linkage disequilibrium was observed for any of the marker loci, including D12S83 ( $\mathrm{p}=0.35$ for the two point linkage test). Multipoint linkage analysis also failed to reveal positive linkage on chromosome 12. Power calculations allowed us to reject the hypothesis that the genetic region of chromosome 12 centred on D12S83 contains a susceptibility locus with a relative risk $\left(\lambda_{\mathrm{s}}\right)$ equal to or greater than 2.0 in these families.

Conclusion-Failure to detect linkage or linkage disequilibrium in these families suggests that the chromosome 12 locus previously reported to be associated with genetic predisposition to IBD does not play a role in all European family samples. This observation is compatible with heterogeneity in the genetic basis of susceptibility to the disease and/or exposure to various environmental factors among Caucasian families.

(Gut 2000;47:787-791)

Keywords: chromosome 12; inflammatory bowel disease; Crohn's disease; linkage analyses; replication study

Inflammatory bowel disease (IBD) includes ulcerative colitis (UC) and Crohn's disease (CD), both of which are multifactorial disorders whose aetiology appears to result from the interaction between environmental and genetic factors. ${ }^{1}$ However, the contributions of genetic background and environmental exposure in the development of the disease are not completely understood. Identification of the relevant environmental factors and susceptibility genes would be an important contribution to the understanding of the pathogenic mechanism involved in IBD.

To date, two susceptibility loci have been mapped on the genome with a non-ambiguous statistical threshold of significance. The first susceptibility locus, named IBD1, was localised in the pericentromeric region of chromosome $16 .^{2}$ This finding has now been confirmed by five independent groups. ${ }^{3-7}$ IBD1 was reported to be involved in the susceptibility to CD but its contribution to UC is still being debated. ${ }^{3-5} 8$

A second locus has been mapped on the long arm of chromosome 12. ${ }^{9}$ This observation has now been reproduced in at least three independent published studies. ${ }^{7011}$ This locus was reported to be involved in the genetic predisposition to both CD and UC. More recently, positive linkage disequilibrium has been reported between this locus and the microsatellite marker D12S83, thus refining the localisation of the predisposing gene. ${ }^{12}$

The aim of this work was to replicate the linkage and linkage disequilibrium on chromosome 12 using an independent set of $170 \mathrm{Cau}-$ casian CD families.

\section{Materials and methods}

PATIENTS AND FAMILIES

One hundred and seventy families affected by CD were recruited through a large European consortium. However, most originated from France $(n=148)$ and Belgium $(n=17)$. Each contributor obtained approval of the relevant ethics committee to participate in the study and informed consent was obtained from each family member.

Diagnostic criteria for inclusion in the study have been published previously and take into account clinical, endoscopic, radiological, and pathological data. ${ }^{13}$ To limit the expected genetic heterogeneity, families containing individuals affected by UC were excluded.

Ninety five families had two or more affected members (multiplex families) and were used for linkage and linkage disequilibrium studies. In total, they included 329 healthy members

Abbreviations used in this paper: $\mathrm{CD}$, Crohn's disease; cM, centiMorgan; HRR, haplotype relative risk; IBD, inflammatory bowel disease; TDT, transmission disequilibrium test; UC, ulcerative colitis; PCR, polymerase chain reaction; NPL, non-parametric linkage. 


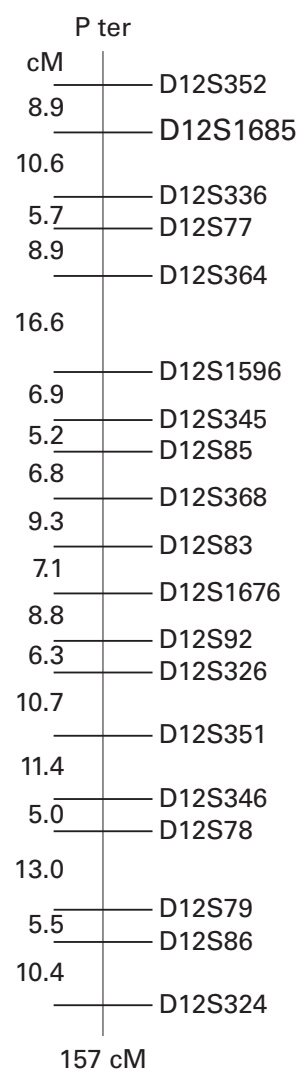

Figure 1 Linkage map of chromosome 12 markers. The genetic map was derived from genotyping data of 170 families with Crohn's disease and generated by the Crimap program. The markers used were selected from the $A B I$ Prism linkage mapping set version 1.0 and from the (http:/www.genethon.fr). Genethon database

and 231 affected relatives accounting for 128 affected sib pairs and 29 affected pairs of second and third degree relatives. In $70 \%$ of families, both parents of affected patients were available; $18 \%$ had only one genotyped parent. For most of the families with a missing parent(s), the parental genotypes could be inferred from the genotypes of the healthy sibs. Thus the number of alleles identical by descent for relative pairs could be inferred in almost all cases.

Seventy five additional families with a single affected member (simplex nuclear families) were also used for association studies. The two parents were available in all cases, making intrafamilial association studies possible.

PROCEDURES FOR GENOTYPING

Genomic DNA was prepared from peripheral blood leucocytes using standard methods. Nineteen polymorphic microsatellite loci were selected from the Applied Biosystems (ABI, Courtaboeuf, France) fluorescent labelled human linkage mapping set version 1.0 (panels $17,18,19)$ or from the Genethon database ${ }^{14}$ and amplified by polymerase chain reaction (PCR) (30 cycles) on an ABI 877 catalyst robotic workstation in $5 \mu \mathrm{l}$ reactions containing $20 \mathrm{ng}$ of genomic DNA, $3 \mathrm{mM} \mathrm{MgCl}_{2}, 0.25$ $\mathrm{mM}$ dNTPs (Pharmacia, Orsay, France), 1.5 pmol of each primer, and 0.4 units of Amplitaq-Gold DNA polymerase (PerkinElmer, Courtaboeuf, France) in $1 \times$ PCR Buffer II (Perkin Elmer).

Pooled amplification products were then subjected to electrophoresis in 5\% polyacrylamide gels for two hours at $3000 \mathrm{~V}$ using an ABI 377 DNA Sequencer. Control DNA (individual 1347-02 from the CEPH reference families) was systematically used as an internal standard. Semi-automated fragment sizing was performed using Genescan 2.1 software (ABI) followed by allele identification using Genotyper 2.0 software (ABI). Each genotype was

Table 1 Two point linkage and linkage disequilibrium analyses

\begin{tabular}{lllll}
\hline & \multicolumn{2}{l}{ Sib pair analysis } & & \\
\cline { 2 - 3 } Markers & $\begin{array}{l}\text { Mean proportion } \\
\text { of alleles shared }\end{array}$ & $p$ Value & $\begin{array}{l}\text { TDT test } \\
\text { pvalue }\end{array}$ & $\begin{array}{l}\text { HRR analysis } \\
p \text { value }\end{array}$ \\
\hline D12S352 & 0.55 & 0.13 & 0.50 & 0.50 \\
D12S1685 & 0.59 & 0.02 & 0.50 & 0.50 \\
D12S336 & 0.50 & 0.50 & 0.50 & 0.50 \\
D12S77 & 0.50 & 0.50 & 0.35 & 0.50 \\
D12S364 & 0.51 & 0.35 & 0.31 & 0.50 \\
D12S1596 & 0.50 & 0.50 & 0.17 & 0.50 \\
D12S345 & 0.50 & 0.50 & 0.50 & 0.50 \\
D12S85 & 0.50 & 0.50 & 0.36 & 0.50 \\
D12S368 & 0.51 & 0.44 & 0.50 & 0.50 \\
D12S83 & $\mathbf{0 . 5 1}$ & $\mathbf{0 . 3 5}$ & $\mathbf{0 . 5 0}$ & $\mathbf{0 . 5 0}$ \\
D12S1676 & 0.51 & 0.35 & 0.26 & 0.50 \\
D12S92 & 0.50 & 0.47 & 0.38 & 0.50 \\
D12S326 & 0.50 & 0.50 & 0.50 & 0.50 \\
D12S351 & 0.50 & 0.50 & 0.17 & 0.50 \\
D12S346 & 0.50 & 0.50 & 0.14 & 0.50 \\
D12S78 & 0.50 & 0.50 & 0.28 & 0.50 \\
D12S79 & 0.52 & 0.26 & 0.04 & 0.50 \\
D12S86 & 0.54 & 0.15 & 0.39 & 0.50 \\
D12S324 & 0.50 & 0.50 & 0.50 & 0.50
\end{tabular}

Results were obtained using 95 multiplex families with Crohn's disease (CD) for the sib pair analysis and $170 \mathrm{CD}$ multiplex and simplex families for the transmission disequilibrium (TDT) and haplotype relative risk (HRR) tests. Analyses were performed with the Analyze package version 3.0. ${ }^{17}$ For the 19 markers of chromosome 12, p values of the linkage (sib pair analysis) and association (TDT and HRR analyses) tests are indicated. The marker D12S83 which previously demonstrated a positive linkage and association ${ }^{71011}$ is in bold. determined independently by two investigators to confirm the accuracy of genotype. Conflicting data were either resolved or discarded.

\section{DATA ANALYSIS}

Mendelian segregation of alleles was checked using the Unknown program version 5.23. Marker ordering and inter-marker distances were computed using the CRI-MAP program. ${ }^{15}$ Any apparent double recombinants were identified and the genotypes rechecked.

Non-parametric multipoint score (NPL) values, information content map, and exclusion maps were produced by the Sibs component of the Genehunter package version 2.0 $0^{16} 17$ using the map generated with the families studied. The NPL statistics measure identity by descent allele sharing between affected relatives. Information content mapping measures the fraction of the total inheritance information extracted from all markers simultaneously. Exclusion maps were constructed assuming no dominance variance and using a locus specific relative risks to siblings $\left(\lambda_{\mathrm{s}}\right)$ value of 2.0 , as previously reported for the chromosome 12 susceptibility locus. A locus was considered to be excluded for linkage if the logarithm of the odds score (LOD score) was less than -2.0 for the specified value of $\lambda_{s}$.

Non-parametric two point analyses were performed using the Analyze package version 3.0. ${ }^{18}$ It includes a two point affected sib pairs analysis that tests for an excess of allele sharing between affected siblings. A search for association between the marker alleles and CD was performed using the transmission/ disequilibrium test (TDT) and the haplotype relative risk analysis (HRR) based on likelihood ratio statistics. Intrafamilial association tests compare the distribution of alleles transmitted and not transmitted to the affected patients from their parents. In the TDT, only data from heterozygous parents are included in the analysis. In the HRR statistic, homozygous parents are also taken into account.

\section{Results}

Members of the $170 \mathrm{CD}$ families were genotyped for 19 highly polymorphic markers, evenly spread on chromosome 12 and including the D12S83 locus that exhibited positive linkage and linkage disequilibrium in previous studies. ${ }^{1012}$ The genetic map generated using these genotypes spanned 157 centiMorgan (cM), a genetic length slightly shorter than the previously estimated length of chromosome 12 (174 cM, see Dib and colleagues ${ }^{14}$ ). The positions of the markers and the inter-marker genetic distances were in agreement with the published maps. The markers were $8.7 \mathrm{cM}$ apart on average (range $5.0-16.6 \mathrm{cM}$ ). As the data from our families were more extensive than previously published, we used the map distances estimated from our data in subsequent statistical analyses (fig 1 ).

Non-parametric two point linkage analyses performed on the 95 multiplex families did not provide evidence of linkage for any of the polymorphic markers tested (table 1). The observed allele sharing between affected siblings 
was maximal at D12S1685 on the short arm of chromosome 12 (observed value 0.59 compared with an expected value of 0.50). However, this locus was $70 \mathrm{cM}$ from D12S83 and was thus far from the genetic region of interest. In the genetic region of interest, centred around the marker D12S83, the maximum value of identity by descent between affected siblings was no more than 0.51 (expected value $0.50 ; \mathrm{p}=0.35$ ) providing no evidence of linkage.

Non-parametric multipoint linkage analyses are shown in fig 2. The information statistic measures the proportion of the possible inheritance information extracted at each location on the map using the panel of polymorphic markers in the families studied (fig 2A). The information is about $80 \%$ on average, indicating that the markers used are highly informative. The multipoint linkage statistic did not indicate linkage at any point on the chromosome (maximum value at $\mathrm{D} 12 \mathrm{~S} 86, \mathrm{NPL}=1.52$, $\mathrm{p}=0.07$ ) (fig 2B). In the genetic region surrounding the D12S83 locus, the NPL
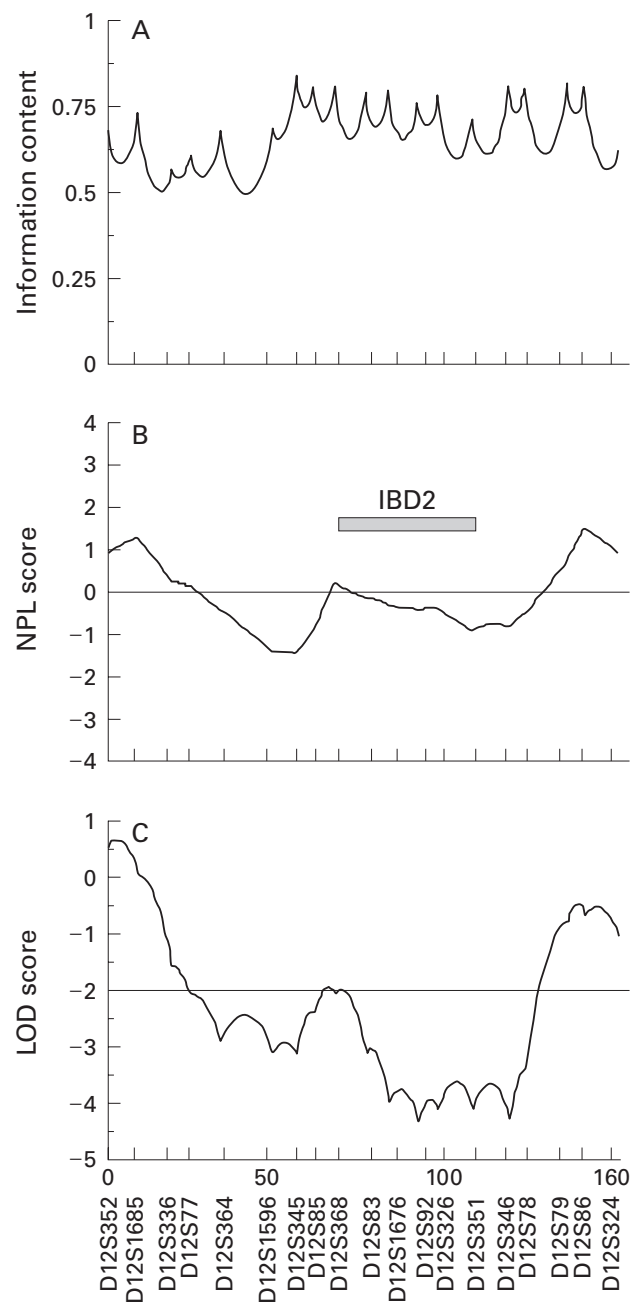

Figure 2 Multipoint linkage analyses of chromosome 12 in 95 multiplex families with Crohn's disease (CD) using the Genehunter package. (A) Information content; (B) multipoint non-parametric linkage (NPL) statistics. The region implicated by Satsangi et al containing the inflammatory bowel disease (IBD) susceptibility locus is indicated by a shaded box. (C) Exclusion map calculated for a hypothetical locus with a relative risk $\lambda=2$. The exclusion threshold of LOD score $(-2)$ is indicated.
Table 2 Results and power of the previously published linkage analyses on chromosome 12 for Crohn's disease (CD) families

\begin{tabular}{|c|c|c|c|}
\hline Author & $\begin{array}{l}\text { No of } \\
\text { multiplex } C D \\
\text { families }\end{array}$ & $\begin{array}{l}\text { No of affected } \\
\text { relative pairs }\end{array}$ & $\begin{array}{l}\text { Results ( } p \\
\text { value of the } \\
\text { test) }\end{array}$ \\
\hline Satsangi et al ${ }^{9}$ & 67 & 81 & $3 \times 10^{-7}$ \\
\hline Duerr $e t a l^{10}$ & 80 & 127 & 0.0002 \\
\hline Yang et $a l^{11}$ & 48 & 75 & 0.0004 \\
\hline Curran et al & 134 & 167 & 0.005 \\
\hline Cho et $a l^{20}$ & 99 & 175 & 0.40 \\
\hline Brant et al $l^{1}$ & 77 & 148 & 0.30 \\
\hline Rioux $e t a l^{2}$ & - & 114 & 0.24 \\
\hline Present study & 95 & 157 & 0.35 \\
\hline
\end{tabular}

values were negative indicating that sharing of alleles identical by descent was less than that expected in this region. Because negative results may arise by chance when statistical power is low, the power to detect linkage using these data was analysed. For a locus specific increased relative risk of $\lambda_{\mathrm{s}}=2$, a value previously attributed to the chromosome 12 susceptibility locus, ${ }^{9}$ the entire genetic region of interest was excluded (fig 2C).

Finally, because linkage disequilibrium was reported between the IBD2 locus and D12S83 by three independent groups, ${ }^{10-12}$ a search for associations was also performed (table 1). Because multiplex and simplex families are informative in association studies, 75 additional simplex families were included in this analysis. Using all of the $170 \mathrm{CD}$ families, it was not possible to demonstrate an association, using either the TDT or HRR test, for any typed chromosome 12 locus

As genetic heterogeneity has already been demonstrated in this disease, we also classified our families into different phenotypic subgroups and repeated the statistical analyses on each group. Neither linkage nor linkage disequilibrium was observed in any of the phenotypic subgroups, including: age at onset below or above 20 years; small intestine and/or colon involvement; or low or high severity during the disease course (data not shown).

\section{Discussion}

The study was performed to determine the contribution of a previously identified IBD susceptibility locus on chromosome 12 in an independent set of Caucasian families.

The initial study reporting linkage between IBD and a susceptibility locus on chromosome 12 used a total of 160 IBD families. In that study linkage was observed for IBD $(\mathrm{p}=0.0000003)$ as well as for UC $(\mathrm{p}=0.0025)$ and $C D$ alone $(p=0.0003) .{ }^{9}$ Even if these results do not argue for a genetic heterogeneity between the two disorders, given the phenotypic differences between CD and UC, genetic heterogeneity in susceptibility cannot be ruled out. For this reason, we decided to limit our investigation to the $\mathrm{CD}$ phenotype. In the original study, linkage for CD was initially found in a group of $67 \mathrm{CD}$ families containing 81 affected sib pairs. ${ }^{9}$ Because the number of families required to replicate a positive linkage may be significantly greater than the number initially used to find linkage, we studied a nearly twofold larger sample of families (95 
families corresponding to 157 affected relative pairs) than that used in the initial study. Our sample was comparable in size to those in other linkage replication studies (table 2). Whereas the initial investigation was performed on affected sibling pairs only, the present study also examined second and third degree relative pairs, which is more informative. Thus these arguments suggest that the panel of CD families studied here was sufficient to detect linkage if it existed in this sample.

The density of markers used in our study was sufficient to extract, on average, $80 \%$ of the inheritance information on the long arm of chromosome 12 using non-parametric multipoint analyses. Furthermore, there is no information gap in the region of interest. Thus it is unlikely that addition of new markers would reveal linkage on this region using the same sample. The linkage tests reported here are model free- - that is, they do not require any assumption about the genetic model underlying the predisposition to CD. Thus the negative results we obtained are not likely to be due to incorrect parameterisation in the statistical calculation. Also, as identity by descent between affected relatives could be inferred unequivocally for most of the relative pairs, it is unlikely that allele frequency errors significantly affected the results.

We were unable to demonstrate linkage between $\mathrm{CD}$ and any locus mapped on chromosome 12 . The linkage score was negative on most of the chromosome and reached its highest positive value of $1.52(p=0.07)$ for a marker located on the short arm of chromosome $12,70 \mathrm{cM}$ away from the linkage peak reported by Satsangi and colleagues. ${ }^{9}$ Considering that it does not correspond to a region with a previously reported linkage, the value of the statistical test $(p=0.02)$ did not reach the threshold of significance widely accepted to demonstrate linkage in a genome wide screen $\left(\mathrm{p}<0.00002\right.$, see Kruglyak and Lander $\left.{ }^{19}\right)$.

In contrast, no linkage was found to the genetic region previously implicated in susceptibility to IBD. To test if this negative result could be attributed to lack of power, an exclusion map was generated. The exclusion map allowed us to conclude that, in our families, no locus with $\lambda_{\mathrm{s}} \geqslant 2$ could be located within the genetic region proposed by Satsangi and colleagues. ${ }^{9}$ However, a gene with a smaller effect cannot be ruled out.

An association between IBD and the microsatellite marker D12S83 has also been reported. ${ }^{12}$ This observation prompted us to replicate this result. Familial association studies have the advantage over case control studies with unrelated controls of avoiding biases due to population stratification. They are thus considered as more reliable for association studies. However, using $170 \mathrm{CD}$ families, we failed to reproduce the reported association in our family panel.

Our findings indicate that in our family panel the IBD locus mapped on chromosome 12 had at most a small effect. Several other studies have also failed to find linkage on the long arm of chromosome 12 (table 2). Only three of seven replication studies detected the IBD locus on chromosome 12. In contrast, four of five replication studies detected the IBD1 locus on chromosome 16.

Several explanations may be proposed to explain the discrepancies between studies. Differences in diagnostic criteria may play a role. In most studies, including ours, the diagnostic criteria used were widely accepted and have been used by numerous investigators. Furthermore, several authors used the same diagnostic criteria and obtained discordant results. ${ }^{79}$ It has been suggested that the chromosome 12 locus might be more important for UC than CD. ${ }^{19}$ However, in most studies reporting a positive linkage, the contribution of the CD families was at least as important as that of the UC families. ${ }^{79}{ }^{10}$ Furthermore, when different phenotypes were analysed separately in the present study, we were unable to detect linkage or linkage disequilibrium. It is thus unlikely that the present negative results are related to major clinical differences.

Differences between statistical analyses could also be considered. However, the statistics used in the above mentioned studies were based on model free methods and were often identical. Thus statistical methods are unlikely to explain the observed discrepancies.

Ethnic heterogeneity may also result in discordant results. All of the published studies were performed in Caucasian families of European origin. They were often the result of a multicentre effort of recruitment in the $\mathrm{USA}^{10}{ }^{19} \mathrm{Canada}^{21}$ or Europe (Curran et $a l,{ }^{7}$ Satsangi et $a l,{ }^{9}$ and the present study). However, ethnic differences cannot be ruled out. For example, it should be noted that the chromosome 12 locus was detected in two cohorts with a large proportion of families recruited in the UK and was less often identified in other geographic samples. It has also been suggested that the IBD susceptibility loci may be different in Ashkenazi Jews. ${ }^{3}$ But discordant results were also obtained in populations with comparable proportions of Ashkenazi Jews in North America. ${ }^{10-21}$

Linkage analyses on the chromosome 16 locus (IBD1) are often available in the family samples used for linkage studies on chromosome $12 .^{34720-22}$ With the exception of the Canadian set of families, all samples also provided evidence of linkage on chromosome 16 (including the present sample, data not shown). Thus linkage on chromosome 12 does not seem to be conditional on linkage on chromosome 16.

Finally, the discrepancy could be explained by the effect of differences in exposure to unknown environmental factors. Indeed, because IBD are multifactorial disorders, a susceptibility gene interacting with a specific environmental risk factor may be more easily discovered in an exposed population. In contrast, in a non-exposed population, the same gene may remain undetected because its contribution to the disease is small. Further investigations, stratifying the genetic analyses for environmental risk factors, are required to highlight this hypothesis. 
This project received financial support from: the European Community (Contract Biomed 2 No BMH4-97-2098), the Ministère Français de l'Education Nationale et de la Recherche, INSERM, the Direction Générale de la santé (convention No 4T004C), the Association François Aupetit, and the Institut de Recherche des Maladies de l'Appareil Digestif. We are grateful to Drs Bruno Bonaz, Yoram Bouhnik, Jean-Pierre Cézard, Salvatore Cucchiara, Jean-Jacques Delchier, Jean-Louis Dupas, Yigael Finkel, Jean-Paul Galmiche, Jean-Pierre Gendre, Denis Golfain, Corinne Gower-Rousseau, Denis Heresbach, André Lachaux, Hervé Lautraite, Catherine Lenaerts, Eric Lerebours, Victor Levy, Robert Löfberg, Helmut Malchow, Rober Modigliani, Colm O'Morain, Francesco Pallone, André Rotenberg, Jacques Schmitz, Iradj Sobhani, André Van Gossum, Myriam Van Winckel, and Michel Veyrac who contributed to family recruitment and to Jeanne Macry from INSERM U458, Isabelle Rousseau from EPIMAD, Jean-Christophe Beaudouin, Christophe Billon, Hung Bui, Lucien Cazes, Catherine Guidicelli, Juliette Langlois, Marie Legrand, Agnès MarcadetTroton, Anna Martins, Catherine Massart, Carole Merlin, Mourad Sahbatou, Eric Poullier, Jean-Marc Sebaoun, Emmanuel Tubacher, and Christelle Vaury from the Fondation Jean Dausset/CEPH for their excellent technical support. We also thank Leigh Pascoe for manuscript revision.

1 Thomas G, Hugot JP. Mapping susceptibility loci for Thomas G, Hugot JP. Mapping susceptibility loci for 33.

2 Hugot JP, Laurent-puig P, Gower-Rousseau C, et al. Mapping of a susceptibility locus for Crohn's disease on chromosome 16. Nature 1996;379:821-3.

3 Ohmen JD, Yang HY, Yamamoto KK, et al. Susceptibility locus for inflammatory bowel disease on chromosome 16 has a role in Crohn's disease, but not in ulcerative colitis. Hum Mol Genet 1996;5:1679-83.

4 Parkes M, Satsangi J, Lathrop GM, et al. Susceptibility loci in inflammatory bowel disease. Lancet 1996;348:1588.

5 Cho JH, Fu Y, Kirshner BS, et al. Confirmation of a susceptibility locus, IBD1, for Crohn's disease on chromosome 16. Inflamm Bowel Dis 1997;3:186-90

6 Cavanaugh JA, Callen DF, Wilson SR, et al. Analysis of Australian Crohn's disease pedigrees refines the localization for susceptibility to inflammatory bowel disease on chromosome 16. Ann Hum Genet 1998;62:291-8.

7 Curran ME, Lau KF, Hampe J, et al. Genetic analysis of inflammatory bowel disease in a large European cohort supports linkage to chromosomes 12 and 16. Gastroenterology 1998;115:1-7.

8 Mirza MM, Lee J, Teare D, et al. Evidence of linkage of the inflammatory bowel disease susceptibility locus on chro- mosome 16 (IBD1) to ulcerative colitis. 7 Med Genet 1998 ; 35:218-21.

9 Satsangi J, Parkes M, Louis E et al. Two stage genome wide search in inflammatory bowel disease provides evidence for susceptibility loci on chromosomes 3, 7 and 12. Nat Genet 1996;14:199-202.

0 Duerr RH, Barmada M, Zhang L, et al. Linkage and association between inflammatory bowel disease and a locus on chromosome 12. Am f Hum Genet 1998;63:95-100.

11 Yang $\mathrm{H}$, Ohmen JD, Ma R, et al. Linkage and association between Crohn's disease and a putative locus on chromosome 12. Gastroenterology 1998;114:A902.

12 Parkes A, Satsangi J, Jewell DP, et al. Transmission disequilibrium of a marker haplotype fine maps chr12 linkage in IBD. Gut 1998;42 (suppl):A49.

13 Lennard-Jones JE. Classification of inflammatory bowel disease. Scand f Gastroenterol 1989;24(suppl 170):2-6.

14 Dib C, Fauré S, Fizames C, et al. A comprehensive genetic map of the human genome based on 5,264 microsatellites. Nature 1996;380:152-4.

15 Lander E, Green P. Construction of multilocus genetic linkage maps in humans. Proc Natl Acad Sci USA 1987;84: 2363-7.

16 Kruglyak L, Daly MJ, Reeve-Daly MP, et al. Parametric and non parametric linkage analysis: a unified multipoint approach. Am f Hum Genet 1996;58:1347-63.

17 Kruglyak L, Lander ES. Faster multipoint linkage analysis using Fourier transforms. F Comput Biol 1998;5:1-7.

18 Terwilliger JD. A powerful likelihood method for the analysis of linkage disequilibrium between trait loci and one or more polymorphic marker loci. Am f Hum Genet 1995;56: $777-87$

19 Kruglyak L, Ander E. Genetic dissection of complex traits: guidelines for interpreting and reporting linkage results. Nat Genet 1995;11:241-7.

20 Cho JH, Nicolae DL, Gold LH, et al. Identification of novel susceptibility loci for inflammatory bowel disease on chromosomes 1p, 3q, and 4q: evidence for epistasis between $1 \mathrm{p}$ and IBD1. Proc Natl Acad Sci USA 1998;95:7502-7.

21 Brant SR, Fu Y, Fields CT, et al. American families with Crohn's disease have strong evidence for linkage to chromosome 16 but not chromosome 12. Gastroenterology 1998;115:1056-61.

22 Rioux JD, Daly MJ, Green T, et al. Absence of linkage between inflammatory bowel disease and selected loci on chromosomes 3, 7, 12, and 16. Gastroenterology 1998;115: $1062-5$

\section{8th United European Gastroenterology Week}

The UEGW abstract book (Gut 2000;47(suppl III)) has again been produced as a CD-ROM and can be found attached to the inside back cover of this issue. 